\title{
Delocalization of two interacting particles in a random potential: one-dimensional electric interaction
}

\author{
J. C. Flores \\ Universidad de Tarapacá, Departamento de Física, Casilla 7-D, \\ Arica, Chile
}

\begin{abstract}
We consider a continuous one dimensional model of two charged interacting particles in a random potential. The electric repulsion is strictly one dimensional and it inhibits Anderson localization. In fact, the spectrum is continuous. The case of electrical attraction is briefly studied and it shows bounded states. So, the dynamics is sign-dependent for this model.We support our analytical results with numerical simulations where the effect of repulsion breaking localization is clearly observed.
\end{abstract}

A complete version of this paper (with figures): Phys.Rev.B 62, 33 (2000). 
The problem of one particle in a random potential has been studied widely after the pioneer work by Anderson [1-5]. Now, it is accepted that exponential localization could take place in such disordered systems depending on the degree of disorder, the dimension $D$, and the energy of the system. The role of internal spatial local correlations at the random potential has been considered as mechanism of delocalization. In fact, there is theoretical [6-19] and experimental [20] evidence of delocalization due to correlations. Moreover, the role of no local correlation has been studied showing new phenomena of delocalization [21]. Also, the dynamics of some open random systems has been studied showing the breaking of Anderson localization [22]. Recently, the role of interaction between particles in disordered systems has been considered (two interacting particles or TIP). Nevertheless, here some controversy exist [23-31]. It seems that for on-site interaction (discrete model), the localization length is enhanced with independence on the interaction sign.

In this paper, and for strictly one-dimensional electric repulsion, we show that Anderson localization is broken for two interacting particles. Moreover, the behavior of the system is not sign-independent. Our quantum model is continuous and different of the usual discrete TIP model found in the literature.

Consider Gauss's theorem in $D$ dimension, namely a charge $q$ producing an electric field $E$ and a hypersphere of radius $r$ around the charge. The theorem establishes that the electric flux through the hypersphere is proportional to the charge enclosed. Namely,

$$
\operatorname{Er}^{D-1} \sim q
$$

So, the electric field produced by a charge is dependent on the dimension $D$. In three dimensions we have the usual $r^{-2}$ dependence and in strictly one dimension the electric field is constant at the left and right of the charge $q$. Namely, in one dimension, the electric potential produced by one charge $q$ is given by

$$
V(x)=-\alpha q|x|, \quad D=1,
$$

where $\alpha$ is a proportionality constant and $x$ the position.

A remark concerning the one dimensional case. Since for a 'sphere' in the one dimension we have problems defining its surface, the result (2) is also obtained by solving the Poisons equation $V^{\prime \prime} \sim q \delta(x)$ with the corresponding 
conditions of continuity $V^{+}(0)=V^{-}(0), \frac{d}{d x} V^{+}(0)-\frac{d}{d x} V^{-}(0) \sim q$ and the symmetry condition $V(x)=V(-x)$.

In order to consider two charged interacting particles in strictly onedimensional disordered potential, we must consider the above electric potential in the Hamiltonian: let $x$ and $y$ be the coordinate-operator of two particles of charge $q$ and $Q$, respectively; then the Hamiltonian-operator of the systems is given by

$$
H=-\frac{1}{2 m} \frac{\partial^{2}}{\partial x^{2}}-\frac{1}{2 M} \frac{\partial^{2}}{\partial y^{2}}+\xi(x)+\xi(y)-\alpha q Q|y-x|,
$$

where $\xi$ denotes the random potential acting on every particle. To study the above system we assume the following

(i) Particles do not overlap. Namely, we assume the charge $Q$ always on the right of $q$.

(ii) Particles are distinguishable $(Q \neq q)$, however by simplicity we assume $M=m$.

(iii) The random potential is bounded $\left(\xi<\xi_{\max }\right)$ and has no internal correlation. Namely, if $\xi(x)=\sum f_{i}\left(x-x_{i}\right)$ then every function $f_{i}$ is independent (also the random variable $x_{i}$ ). So, if there are extended states they are not related to correlations at the random potential [6-19].

(iv) No decoherence effects due to an external bath, or measurement, are considered i.e. we have always a quantum pure state.

Let the state $\Psi_{\omega}(x, y)$ be solution of the time independent Schrödinger equation, namely

$$
H \Psi_{\omega}=\omega \Psi_{\omega},
$$

where $\omega$ is the energy $(\hbar=1)$. The condition (i) requires that

$$
\Psi_{\omega}(x, y)\left\{\begin{array}{l}
=0 ; y \leq x \\
\neq 0 ; y>x
\end{array} .\right.
$$

Since the charge $Q$ is on the right of $q$, the electrical interaction does not require the absolute value i.e. $|y-x|=y-x$. The Schrödinger equation (4) becomes separable, and related to the solution of 


$$
-\frac{1}{2 m} \frac{\partial^{2}}{\partial z^{2}} \phi+(\xi(z) \pm \alpha q Q z) \phi=\epsilon \phi,
$$

where the constant $\epsilon$ is the energy. Since we are assuming electrical repulsion $(Q>0, q>0)$, the sign minus (or plus) in (6) corresponds to the equation for $Q$ (or $q$ ). So, the charge $Q$, on the right of $q$, experiences two forces: the random due to disorder, and the electrical repulsion directed to the right. The charge $q$ is repelled to the left.

The continuous Schrödinger equation (6) has been studied in the literature and it corresponds to a particle in a disordered potential with a dc electric field. For bounded disordered potential (iii), the states $\phi$ are extended in the direction of the field and the spectrum $\epsilon$ is continuous [32-35]. This can be understood intuitively when we consider that for a distance $|z| \gg \xi_{\max } / \alpha q Q$ the electric dc field dominates the asymptotic behavior of the system and producing extended states. We remark that our model is continuous, with bounded disorder, as required by the theory of delocalization with electric dc field [32].

In this way, the solution of equation (4) can be written as

$$
\Psi_{\omega}(x, y)=\left\{\phi_{\epsilon}(x) \phi_{\omega-\epsilon}(y)-\phi_{\epsilon}(y) \phi_{\omega-\epsilon}(x)\right\} ; x \leq y,
$$

where $\phi_{\epsilon}$ denotes the extended solution of energy $\epsilon$ of (6). We remark that the condition (i) (not overlap $\Psi(x, x)=0$ ) is satisfied automatically from the antisymmetric condition for the wave function. Then, for repulsion, localization is inhibited for two charged interacting particles in strictly one dimension. Moreover, the spectrum $\omega$ is continuous and determined asymptotically by the electric interaction. Namely, no periodic (or quasiperiodic) motion exists in the system.

Numerical time-evolution calculations confirm our conjecture about delocalization in the repulsive case: Figure (1a) shows the time-dispersion for two wavepackets (particles) when interaction and disorder do not exist. The initial condition is the localized state $\Psi(x, y, t=0)=\delta_{x, x_{o}} \delta_{y \cdot y_{o}}$ and the regime is ballistic. It shows the probability $\int d x|\Psi(x, y, t)|^{2}$ and $\int d y|\Psi(x, y, t)|^{2}$ to find the particle $Q$ and $q$ respectively at time $t \neq 0$. Figure (1b) shows the two particles under one-dimensional electric repulsion and the same initial conditions of (1a). Remark the time separation of the center of mass for every particle because repulsion. Figure (1c) shows the evolution when only disorder is present, and one notes the absence of dispersion for the wavepackets. 
Finally, Figure (1d) shows the combined effect of repulsion and localization. Clearly, disorder does not stop the separation due to repulsion of both particles. The numerical calculations were carried-out by solving the Schrödinger equation related to (3) with a simple procedure (finite-differences). The potential $\xi$ was constructed using a random number generator. The spatial boundary was avoided by considering a finite number of iterations. This number was found by using the iteration procedure related to figure (1b), the most fast motion. All figures have the same parameters (number of iterations, step, disorder, etc.).

On the other hand, when electrical attraction is considered in our onedimensional systems, the numerical results show the existence of bounded states. Figures (2a) and (2b) show the temporal evolution for two interacting particles in a random potential. (2a) shows the repulsive behavior related to delocalization. Figure (2b) shows the evolution when one changes the parameter of repulsion by attraction and keeping the same initial conditions and disorder $(q Q \rightarrow-q Q)$. Clearly the behavior between repulsion and attraction is different. Since we are assuming attraction between both particles, the charge $Q$ is pushed by the field to the left against $q$; but from (i) the wave function vanishes when $y=x$. From an analytical point of view, our result can be understood when we consider no disorder and the usual coordinate change to the centre of mass. Namely $2 X=x+y$ and the relative coordinate $2 r=x-y$. The non overlap condition at $r=0$ and the constant attractive electric field gives origin to bounded states [36]. The finite disorder $\left(\xi<\xi_{\max }\right)$ does not change the behavior of the system governed asymptotically by the electric field. Discrete spectrum and bounded states exist in the system in this case.

Finally, we notice that the one-dimensional electric case studied here is formally similar to two infinite parallel planes separated by a distance $|y-x|$ where the electric force is constant. This tells us that plane charged molecules are good candidates to test our result. Also quantum-wire, with one-dimensional directed electric-flux, seems an interesting candidate to explore our conjectures.

In conclusion: we have presented theoretical evidence that strict one dimensional electrical repulsion, which is long-range, breaks Anderson localization for two interacting charged particles. This result is different to the discrete on-site interaction which only enhances the localization length, but does not break localization. In our case, the attraction and repulsion behavior are sign-depending. These results were confirmed numerically. 
I thank Professor S. N. Evangelou who explain us the TIP Problem (International Workshop on Disordered Systems with Correlated-Disorder, Arica'98, PELICAN and FDI-UTA Projects). Interesting comments were furnished by Professor Chumin Wang Chen, Professor V. Bellani (FDI-UTA and PELICAN Projects) and Professor E. Lazo (UTA). This work was partially completed at Pavia University (CICOPS Scholarship), and partially supported by FONDECYT (proyecto 1000439).

\section{References}

[1] P. W. Anderson, Phys. Rev. 109, 1492 (1958).

[2] E. Abrahams, P. W. Anderson, D. G. Licciardello, and T. V. Ramakrishnan, Phys. Rev. Lett. 42, 673 (1979).

[3] N. F. Mott and W. D. Twose, Adv. Phys. 10, 107 (1961).

[4] K. Ishii, Prog. Theor. Phys. Suppl. 53, 77 (1973).

[5] B. U. Felderhof, J. Stat. Phys. 43, 267 (1986). And references therein.

[6] J. C. Flores, J. Phys. Condens. Matter 1, 8471 (1989).

[7] D. H. Dunlap, H.-L. Wu and P. Phillips, Phys. Rev. Lett. 65, 88 (1990).

[8] H.-L. Wu and P. Phillips Phys. Rev. Lett. 66, 1366 (1991); Science 252, 1805 (1991).

[9] A. Bovier, J. Phys. A 25, 1021 (1992).

[10] J. C. Flores and M. Hilke, J. Phys. A 26, L1255 (1993).

[11] P. K. Datta, D. Giri, and K. Kundu, Phys. Rev. B 47, 10727 (1993); 48, 16347 (1993).

[12] E. Diez, A. Sánchez and F. Domínguez-Adame, Phys. Rev. B 50, 14359 (1994); 50, 17736 (1994).

[13] A. Sánchez. F. Domínguez-Adame, G. Berman, and F. Izrailev, Phys. Rev. B 51, 6769 (1995). 
[14] A. Sánchez, F. Dominguez-Adame. and E. Maciá, Phys. Rev. B 51, 173 (1995); 49, 147 (1994).

[15] S. N. Evangelou and E. N. Economou, J. Phys. A 26, 2803 (1993).

[16] M. Hilke, J. Phys. A 27, 4773 (1994).

[17] J. Heinrichs, Phys. Rev. B 51, 5699 (1995).

[18] B. Lindquist and R. Riklund, Phys. Stat. Sol. (b) 209, 353 (1998).

[19] F. M. Izrailev and A. A. Krokhin, Phys. Rev. Lett 82, 4062 (1999).

[20] V. Bellani, E. Diez. R. Hey, L. Toni. L. Tarricone, G. B. Parravicini, F. Domínguez-Adame, and R. Gomez-Alcalá. Phys. Rev. Lett. 82, 2159 (1999).

[21] F. A. B. F de Morua and M. L. Lyra, Phys. Rev. Lett. 81, 3735 (1998).

[22] J. C. Flores, Phys. Rev. B 60, 30 (1999).

[23] D. L. Shepelyansky, Phys. Rev. Lett. 73, 2607 (1994).

[24] Y. Ymry, Europhys. Lett. 30 , 405 (1995).

[25] K. Frahm, A. Müller-Groeling, J.-L. Pichard, and D. Weinmann, Europhys. Lett. 31, 169 (1995).

[26] D. Weinmann, A. Müller-Groeling, J.-L. Pichard, and K. Frahm, Phys. Rev. Lett. 75, 1598 (1995).

[27] F. von Oppen, T. Wetting, and J. Muller, Phys. Rev. Lett. 76, 491 (1996).

[28] Ph. Jacquod and D. L. Shepelyansky, Phys. Rev. Lett.78, 4986 (1997).

[29] R. A. Römer and M. Schreiber, Phys. Rev. Lett. 78, 515 (1997).

[30] K. Frahm, A. Müller-Groeling, and J.-L. Pichard, Phys. Rev. Lett. 76, 1509 (1996).

[31] P. H. Song and D. Kim, Phys. Rev. Lett. 56, 12217 (1997).

[32] F. Delyon, B. Simon, B. Souillard, Phys. Rev. Lett. 52, 2187 (1984) 
[33] F. Bentosela, R. Carmona, P. Duclos, B. Simon, B. Souillard and R. Weber, Commun. Math. Phys. 88, 387 (1983).

[34] G. V. Vijayagovindan A. M. Jayannavar, N. Kumar, Phys. Rev. B 35, 2029 (1987).

[35] J. C. Flores, H. R. Jauslin and C. P. Enz, J. Phys.:Condens. Matter 1, 123 (1989).

[36] L. Landau and E. M. Lifschitz, Mécanique Quantique Vol III. Editions MIR (1975). 


\section{FIGURE CAPTION}

Figure 1. (1a) Time evolution probability for two particles without disorder and electric interaction. The initial condition is $\Psi(x, y)=\delta_{x, x_{0}} \delta_{y . y_{o}}$. The dispersion is ballistic. For simplicity we do not consider antisymmetric states. (1b) Time evolution probability with electric field repulsion. The parameters are the same as in figure (1a). Still we have no disorder. Remark the increasing separation between the wavepackets with time. (1c) The same situation as in figure (1a) but with disorder and without electric interaction. Clearly, diffusion does not exist because Anderson localization. (1d) Combined influence of electric repulsion and disorder. Disorder does not stop the repulsion process and delocalization operates.

Figure 2. (2a) shows the repulsion for two electric charges in a random potential. Figure $(2 \mathrm{~b})$ shows the attractive case with the same parameter

of disorder and initial conditions. Clearly the behavior is different in both cases. 